\title{
Certain Generalized Quantum Simpson's and Quantum Newton's type Inequalities for Convex Functions in Quantum Calculus
}

\author{
Muhammad Aamir Ali $^{1}$, Hüseyin BUDAK ${ }^{2}$, PRAVEEN AGARWAL ${ }^{3}$, and Yuming Chu ${ }^{4}$ \\ ${ }^{1}$ Nanjing Normal University School of Mathematical Sciences \\ ${ }^{2}$ Düzce University \\ ${ }^{3}$ Anand International College of Engineering \\ ${ }^{4}$ Huzhou University
}

November 25, 2020

\begin{abstract}
In this paper first we present some new identities by using the notions of quantum integrals and derivatives which allows us to obtain new quantum Simpson's and quantum Newton's type inequalities for differentiable convex functions by using the q- $\{x\}-$ quantum integral and $\mathrm{q}^{\wedge}\{\mathrm{y}\}$-quantum integral. In particular, this paper generalises and extends previous results obtained by the various authors in the field of quantum and classical integral inequalities.
\end{abstract}

\section{Hosted file}

MMAS11242020.pdf available at https://authorea.com/users/330633/articles/495090-certaingeneralized-quantum-simpson-s-and-quantum-newton-s-type-inequalities-for-convex-

functions-in-quantum-calculus 